\title{
Theoretical and methodological consequences of variations in exposure duration in visual laterality studies
}

\author{
JUSTINE SERGENT \\ McGill University, Montreal, Quebec, Canada
}

\begin{abstract}
The visual system does not instantaneously extract the entire content of a stimulus, and its resolving power is enhanced as the energy in the stimulus increases. This property of the visual system has been overlooked in previous tachistoscopic studies, the consequences of which are examined. Three groups of 12 right-handed males were presented with faces to categorize as "female" or "male," each group at different exposure duration, 40, 120, or 200 msec. A shift in visual field superiority was observed, from the left to the right field, as stimulus energy increased (Experiment 1). This result was replicated in Experiment 2, using a within-subject design. In Experiment 3, display energy was kept constant by reciprocally varying the duration of exposure and the level of luminance. The same shift in visual field superiority as in the previous experiments obtained when exposure duration increased. Implications of these results for future tachistoscopic studies and for a model of cerebral lateralization are discussed. It is suggested that the right and left hemispheres may not require an equal amount of energy to efficiently engage into cognitive processing.
\end{abstract}

The understanding of cerebral lateralization of cognitive functions in normal adults is no longer solely inferred from research with brain-damaged patients; it now also relies on specific techniquesmainly dichotic listening and lateral tachistoscopic presentations-whose use has proliferated in the last two decades. Early findings from such experiments (Bryden, 1965; Kimura, 1961) proved to be in concordance with evidence obtained from brain-damaged patients, but the greater availability of normals and the possibility of carrying out more complex tasks with normals than with patients have helped to make the use of these techniques one of the major sources of information about functional hemispheric asymmetries.

The use of tachistoscopic presentations in the study of hemispheric asymmetry is appropriate because of the anatomical property of the visual system whereby temporal hemiretinae project to the visual cortex ipsilaterally while nasal hemiretinae project contralaterally. Thus, stimuli presented in the right visual field (RVF) have direct access to the left hemisphere (LH) and stimuli presented in the left visual field (LVF) are directly projected to the right hemisphere ( $\mathrm{RH})$.

This research was partially supported by grants from the Natural Sciences and Engineering Research Council of Canada and from the Quebec Ministry of Education. I am indebted to C. W. Eriksen and two anonymous reviewers for their helpful comments and suggested revisions. The author's mailing address is: Department of Psychology, McGill University, 1205 Docteur Penfield Avenue, Montreal, Quebec H3A 1B1, Canada.
However, two conditions must be met in tachistoscopic studies to ensure that information from one visual field is initially projected only to the contralateral hemisphere: (1) the stimulus must be presented to the right or left of fixation, and (2) the duration of exposure must be short enough-150 msec or less-to prevent eye movements that could expose the stimulus to both hemispheres. ${ }^{1}$

These two conditions are the sine qua non in the use of tachistoscopes in studying hemispheric asymmetry. The values given these two factors extend from almost 0 to $10 \mathrm{deg}$ or more in retinal eccentricity, and from about 5 to $150 \mathrm{msec}$ (but see Footnote 1) in exposure duration. However, these two conditions have been met without consideration of their effects on another property of the visual system. There is perceptual, psychophysical, and neurophysiological evidence that the visual system does not instantaneously extract the entire content of a stimulus, and that more information becomes available as exposure duration increases, as luminance is enhanced, and as retinal eccentricity decreases.

Flavell and Draguns (1957), in a review on the "microgenesis" of perception, reported findings suggesting that "perception is a developmental process consisting of a number of conceptually distinct phases" (p. 198). For example, when a stimulus is presented under conditions of gradually increasing clarity consequent to an increase in exposure duration, the inital perception is that of a diffuse, undifferentiated whole, which then achieves figure-ground discrimination while the inner contents remain vague 
and amorphous; the next stage consists of a greater distinctiveness of contour and inner content; finally, a complete representation is achieved with added elaborations of the "skeletal gestalt" perceived in the previous stage. Flavell and Draguns further cited findings of the same developmental process when stimuli were gradually moved from the extreme periphery of the visual field in towards a central fixation point and brightness was progressively enhanced. As noted by the authors, however, many of these studies would be considered quite poor by presentday methodological standards, as they relied mainly on subjects' introspective reports and drawings of their perceptions. Nonetheless, these findings were obtained in several independent laboratories, and they may indicate that experimental factors such as exposure duration, luminance, and retinal eccentricity influence the quality and completeness of the visual representation achieved in the brain.

The visual system owes its great sensitivity in large part to its ability to integrate luminous energy over time. This integration of energy is described by Bloch's law, which states that visual response is dependent on a reciprocal relationship of time and intensity over a critical duration. For brightness discrimination, the time interval over which complete reciprocity holds is on the order of $100 \mathrm{msec}$. For form perception and spatial acuity tasks, the time intensity reciprocity has a critical duration of 300 400 msec (Kahneman, 1964; Kahneman \& Norman, 1964). Thus, visual acuity develops over time and, as pointed out by Eriksen and Schultz (1978), fine details become discernible later as energy is summed sufficiently to resolve the higher acuity requirements for these details. Riggs (1971, p. 304) suggested that for a test target to be seen with maximum acuity, the exposure duration must be greater than the critical duration.

A large amount of evidence supporting these findings has accumulated since Campbell and Robson (1968) suggested that the visual system contains several independent channels tuned to different bands of spatial frequency. For example, Breitmeyer (1975) and Tolhurst (1975), using gratings of different spatial frequency, found that, in the detection of a threshold grating, reaction time (RT) was much shorter when the grating had low spatial frequency than when it had high spatial frequency, even when the gratings were matched for subjective contrast. Thus, Breitmeyer (1975) observed that RT increased by about $80 \mathrm{msec}$ when spatial frequency increased from .5 to $11 \mathrm{cpd}$ of visual angle. Harwerth and Levi (1978) obtained similar results with suprathreshold gratings. Moreover, Breitmeyer and Ganz (1977), in studying the visual system's temporal response to flashed gratings of variable spatial frequency whose average luminance was $5.4 \mathrm{~mL}$, observed that the critical duration at and below which contrast-duration reciprocity holds increased with spatial frequency, suggesting a briefer integration time for low than for high frequencies. While both low and high frequencies could be detected at 20-msec exposure, more than 20 times higher contrast was required for detecting the high-frequency gratings. As noted by Breitmeyer and Ganz (1977), contrast sensitivity at moderate-to-high spatial frequencies benefits more from increases in exposure duration than does contrast sensitivity at low-to-moderate spatial frequencies, suggesting that the resolving power of the visual system increases with an increase in stimulus energy.

This differential rate of extraction and integration of low and high frequencies may then account for the gradually increasing clarity of perception as exposure duration or luminance increases, as reported by Flavell and Draguns (1957). The early diffuse perception may result from the initial availability of low frequencies; with longer exposure, higher frequencies become available, allowing for a better distinction of the inner contents. It is noteworthy that experiments on detection and integration time of spatial frequencies presented outside the fovea have yet reported no difference between the LVF and the RVF (e.g., Berkley, Kitterle, \& Watkins, 1975; Di Lollo, 1981; Lie, 1980; Rijsdijk, Kroon, \& van der Wildt, 1980), whereas differences between the upper and lower visual fields have been found (Rijsdijk et al., 1980). This may indicate similar extracting and integrating processes in the sensory areas of the right and left hemispheres.

These perceptual and psychophysical properties may have their neural substrates in specific physiological properties of the visual system whose pathways from the retina to the visual cortex are regarded as a group of parallel channels contributing distinctively to vision (see Lennie, 1980, and Stone, Dreher, \& Leventhal, 1979, for reviews). Two main classes of neurons, which possess specific response properties to spatial and temporal characteristics of the stimulus, have been described. Transient neurons respond best to large low-frequency patterns at the onset and offset of a stimulus with brief latency, whereas sustained neurons respond best to small high-frequency patterns of prolonged duration, with long latency (Fukuda \& Stone, 1974; Wright \& Ikeda, 1974). Neurons selectively tuned to narrow spatialfrequency bands have been found in the striate cortex of the cat (Maffei \& Fiorentini, 1977; Tootell, Silverman, \& DeValois, 1981) and the monkey (Schiller, Finlay, \& Volman, 1976). ${ }^{2}$ The existence of such neurons in humans has been suggested by Williamson, Kaufman, and Brenner (1978), who recorded the neuromagnetic field produced by flowing electric current in the visual cortex in response to gratings of varying spatial frequency. They observed 
that latencies of the magnetic response of the striate cortex were highly correlated with the RTs to detect gratings of different spatial frequencies reported by Breitmeyer (1975). The perceptual and psychophysical properties outlined above may then directly derive from these physiological properties of the visual system which underlie the typical variations in spatial-frequency extraction as a function of stimulus energy. ${ }^{3}$

These characteristics of the visual system have not been taken into consideration in tachistoscopic studies, and this may, in fact, be one aspect of a more fundamental omission. The visual stimuli used in these studies are generally described as verbal or visuospatial, such as letters or faces, digits or nonsense shapes, and these represent conceptual categories that are assumed to grasp the essence of the stimuli, but what do they imply in terms of cerebral process?

The early unconscious processing that takes place from the retina to the striate cortex does not differentiate between, for instance, a letter and a face in terms of verbal or visuospatial characteristics. Rather, it is concerned with the physical characteristics of the stimuli, such as edges, line orientations, and spatial frequencies whose neural correlates represent the basic relevant units in visual cerebral processing, and whose extraction and integration during this early processing leads to the emergence of a percept. Thus, cerebral asymmetry in visual information processing is likely to be better understood if it is examined in terms of neural inputs that are processed in the sensory areas of the brain and then transmitted for further processing. In fact, by focusing on abstract categories instead of units that are of relevance in cerebral processes, one has overlooked factors which could influence the characteristics of these units. ${ }^{4}$ Indeed, a letter presented during $5 \mathrm{msec}$ or during $150 \mathrm{msec}$ is the same letter, but its physical characteristics, in terms of spatial frequency contents, may not be the same at both exposures, and neural cells are sensitive to such differences. As a result of this omission, exposure durations and levels of luminance in visual laterality studies have been determined without justification, if not at random, and it is not surprising that many inconsistencies have been emerging in the field of tachistoscopic experiments.

When one examines the findings of these studies by taking into consideration the duration of exposure and the level of luminance, two characteristics may appear noteworthy. First, the value of luminance is seldom reported, and experiments in which exposure duration was manipulated kept luminance constant. This implies that, due to Bloch's law, brightness covaried with variations in exposure duration. Second, a fairly consistent pattern of re- sults seems to emerge as a function of exposure duration: The shorter the exposure duration, the more likely is an LVF-RH superiority effect to appear; the longer the exposure duration, the more likely is an RVF-LH advantage to prevail, and this pattern seems to be independent of the verbal or nonverbal nature of the stimuli. For example, Hellige and Webster (1981) used a 10-msec exposure, 1-mL luminance, and 6-deg-retinal eccentricity to examine laterality effects in the name-identity condition (Aa) of the Posner paradigm. Contrary to the usual, expected RVF superiority (e.g., Cohen, 1972; Davis \& Schmit, 1973), Hellige and Webster obtained an LVF advantage in this condition on 2 consecutive days. Bradshaw, Hicks, and Rose (1979) observed an LVF superiority in a lexical discrimination task when the stimuli were presented for $20 \mathrm{msec}$, but an RVF advantage at longer exposures. This finding was recently replicated by Pring (1981). Rizzolatti and Buchtel (1977) obtained an amplification of the LVF superiority effect, in their male subjects, when the exposure of faces to be recognized was reduced from 100 to 20 msec.

On the other hand, when stimuli that are usually held to be better processed by the $\mathrm{RH}$, such as faces, are presented for a longer duration than usual, one may observe an RVF advantage. Jones (1980), using a 200-msec exposure, obtained an RVF superiority in his right-handed male subjects in the categorization of faces as "female" or "male." Sergent (1982a) found the same RVF advantage in the discrimination of faces that differed in one feature and were presented for $250 \mathrm{msec}$. Marzi and Berlucchi (1977) obtained an RVF superiority with a 400-msec exposure in the identification of well-known faces, whereas a basically similar task yielded an LVF superiority when faces were presented for only $60 \mathrm{msec}$ (Leehey \& Cahn, 1979).

Such variations in field superiority as a function of the luminous energy determined by the duration of exposure could then reflect a differential sensitivity of the hemispheres to spatial frequencies, beyond the sensory areas. Because of the temporal summation of energy, and since during the first milliseconds of stimulus presentation the percept being resolved is essentially a function of the low spatial frequency spectral components, the observed LVF advantage with very brief presentations may result from a better ability of the RH to deal with low frequencies. Conversely, since longer exposure duration benefits the extraction of higher frequencies and yields an RVF superiority, the LH may prove better adapted to processing high frequencies.

Confirmation of this differential sensitivity of the $\mathrm{LH}$ and RH to high and low frequencies may come from a study in which letters of similar shape, complexity, and familiarity, but of differential spatial 
frequency, were presented for $150 \mathrm{msec}$ in a visual search paradigm (Sergent, 1982b). Low-frequency letters were more rapidly recognized in the LVF than in the RVF, whereas the opposite held true for highfrequency letters, independent of variations in exposure duration and luminance. This finding was taken as an indication that the LH may be preferentially, though not exclusively, sensitive to highfrequency information, while the RH could be better adapted to dealing with low frequencies.

\section{EXPERIMENT 1}

This experiment was designed to further examine the hypothesis that the cerebral hemispheres are sensitive to different ranges of spatial frequency. Specifically, it was intended to investigate how variations in stimulus energy, obtained by manipulating exposure duration alone and keeping luminance constant, as in most tachistoscopic studies, would influence the emergence of hemispheric superiority. In fact, the variations in visual field advantage as a function of exposure duration, observed in previous experiments and described above, may have resulted from some other factors not readily controllable, since the studies mentioned were conducted in different laboratories. It would then prove more reliable to investigate the effects of exposure duration on hemispheric asymmetry with the same stimuli and experimental conditions throughout the different exposure durations.

Faces were used as stimuli, primarily because variations in field superiority as a function of exposure have already been reported with letters (Bradshaw et al., 1979; Pring, 1981). In addition, the use of faces was intended to further test the deeply rooted tradition that holds that the RH is specialized in processing faces. In this experiment, subjects were asked to categorize faces as "female" or "male," with RT as the main dependent variable and exposure durations of 40,120 , and $200 \mathrm{msec}$. A shift in visual superiority, from the LVF to the RVF, was expected as stimulus energy increased.

Only males were used as subjects. The purpose of this experiment was thought better served by avoiding the inclusion of the variable "sex" in view of the controversy about the degree of lateralization in women (see Kinsbourne's, 1980, commentary on McGlone, 1980).

The stimuli were presented in the LVF and RVF, as well as in the center of the visual field (CVF). The CVF presentations were aimed at obtaining RT data for comparison of performance at the three different exposure durations.

\section{Method}

Stimuli and Apparatus. The stimuli were 20 front-view photographs of real faces, 10 female and 10 male, borrowed from a local TV station and unknown to the subjects, as assessed by 15 students not involved in the experiment (and confirmed by the subjects after the experiment). Each photograph was transferred on three different slides, on the right side, center, or left side, and was either reduced or enlarged so that all the photographs had about the same size. There were thus 60 slides in all, printed as positives and appearing in black and white on a white background. The stimuli were rear-projected onto a rectangular translucent screen, $18 \times 13 \mathrm{~cm}$, with a permanent black dot located in its center. The faces, when projected, subtended a visual angle of about $3 \mathrm{deg}$ in height and about $2 \mathrm{deg} 20 \mathrm{~min}$ in width. The center of the laterally presented faces was $2 \mathrm{deg} 10 \mathrm{~min}$ from fixation. Another set of 5 female and 5 male faces was similarly prepared for the practice session. The fixation field was set at $3 \mathrm{~mL}$, as was the test field. Viewing was binocular.

The slides were placed in a Kodak random-access projector. Presentation was controlled by a PDP-11/20 computer, which selected the slides in a random order. Each slide appeared four times, yielding a total of 240 experimental trials, with an equal number of female and male faces in each visual field. When a subject made an error, that slide was presented again later in the sequence. Exposure duration, recording of RTs, and an intertrial duration of $4 \mathrm{sec}$ were controlled by the PDP-11/20 computer. Presentation was made through a Lafayette Model 43016 shutter installed on the Kodak projector. The shutter's rise time to full open and closure from full open was $2 \mathrm{msec}$, insuring a relatively square-wave onset and of fset.

Procedure. The subjects were assigned randomly to one of three exposure-duration conditions, and were tested individually. Each subject sat about $80 \mathrm{~cm}$ in front of the screen, his head adjusted in a chin- and foreheadrest so that his eyes were constantly at the level of the central fixation point. The subject's hands rested on a response panel, with the right index finger on one Morse key and the left index finger on another. The keys were placed along the midline axis, one ahead of the other. Within each exposure condition, half the subjects used the right finger if the face was a female and the left finger if the face was a male; this procedure was reversed for the other half.

A 500 -msec tone warned the subject to fixate the central point. The stimulus appeared $1 \mathrm{sec}$ after the onset of the warning tone, and the subject was to press one of the two keys, depending on the sex of the face. The durations of the face presentations were $40 \mathrm{msec}$ for 12 subjects, $120 \mathrm{msec}$ for another 12 subjects, and $200 \mathrm{msec}$ for a third 12 . The subjects were told to respond as quickly and accurately as possible. Each subject was tested in one experimental session of 240 trials, preceded by a practice session of 30 trials. An entire experimental session lasted about $25 \mathrm{~min}$.

Subjects. Thirty-six male students participated in the experiment (age ranging from 19 to 29 years). They were all righthanded, as assessed with Oldfield's (1971) questionnaire, with no history of familial sinistrality, and all had normal or correctedto-normal vision.

\section{Results and Discussion}

A preliminary analysis indicated no reliable effects related to hand responding and to the sex of the faces, and the results were collapsed across these variables. The error rates were $5.8 \%$, and no significant main effects and interaction of condition and visual field was obtained, although there was a trend for more errors in the $40-\mathrm{msec}$ condition. However, this error rate excluded two subjects, in the 120 -msec condition, who repeatedly responded "female" to one particular male face.

A first analysis of variance examined the effects of exposure duration on RTs in CVF presentations. 
RTs significantly decreased as exposure duration increased $[F(2,33)=8.47, p<.002]$. Because of the between-subject's design, the Tukey method was used for pairwise comparisons, and the critical value of $Q(3,33), 1-\alpha=.99$ was 4.42 . RTs were significantly longer at $40-\mathrm{msec}$ than at 120 -msec exposure $(\mathrm{Q}=4.56)$, but $\mathrm{RTs}$ at 120 -msec exposure were not significantly different from RTs at 200 -msec exposure $(Q=.87)$. These results suggest that speed of processing increased as the stimulus energy increased.

A two way analysis of variance was then performed on RTs in lateral visual fields, with one independent factor, exposure, and one repeated factor, visual field. These RTs are shown in Table 1 along with RTs in CVF presentations. The main effect of exposure was significant $[F(2,33)=9.10, p<.001]$, showing a decrease in RT as exposure duration increased. The interaction of exposure $\times$ visual field was also significant $[F(2,33)=22.82, p<.001]$. RTs were shorter in LVF than in RVF presentations at $40-\mathrm{msec}$ exposure but equal at 120 -msec exposure, while RTs were shorter in RVF than in LVF presentations at $200 \mathrm{msec}$. At $40 \mathrm{msec}$, all subjects showed a pattern of results similar to the general trend, and at $200-\mathrm{msec}$ exposure, 11 subjects did so. At $120 \mathrm{msec}, 8$ subjects had RT differences between the two fields inferior or equal to $5 \mathrm{msec}, 3$ subjects responded more quickly to RVF presentations, and 1 subject more quickly to LVF presentations.

Pairwise comparisons to examine the simple effects of this interaction were performed using the Tukey method. The critical value of $Q(6,33), 1-\alpha=$ .99 was 5.20 , and the observed values of $Q$ are shown in Table 2. The RT difference between LVF and RVF presentations at $40-\mathrm{msec}$ exposure was significant $(Q=7.95)$, as was the $R T$ difference between the two visual fields at $200-\mathrm{msec}$ exposure $(Q=6.81)$. The effect of exposure duration on performance within each visual field was then examined. In LVF presentations, the RT difference between 40 - and 120 -msec exposures was significant $(Q=38.63)$, but that between 120 - and 200 -msec exposures was not $(Q=4.54)$. In RVF presentations, RTs significantly decreased between 40 - and 120 -msec exposure $(Q=46.59)$; the decrease in RTs was also significant when exposure increased from 120 to $200 \mathrm{msec}(\mathrm{Q}=11.36)$. Thus, while performance in RVF presentations constantly improved as exposure duration increased, performance in LVF presentations was significantly better

Table 1

Mean Latencies in the Three Visual Fields for Each Exposure Duration of Experiment 1

\begin{tabular}{cccc}
$\begin{array}{c}\text { Exposure } \\
\text { Duration }\end{array}$ & LVF & RVF & CVF \\
\hline 40 & 568 & 582 & 568 \\
120 & 500 & 500 & 495 \\
200 & 492 & 480 & 481 \\
\hline
\end{tabular}

Table 2

Observed Values of $Q$ in the Pairwise Comparisons of Experiment 1

\begin{tabular}{|c|c|c|c|c|c|c|c|}
\hline & & \multicolumn{2}{|c|}{$40 \mathrm{msec}$} & \multicolumn{2}{|c|}{$120 \mathrm{msec}$} & \multicolumn{2}{|c|}{$200 \mathrm{msec}$} \\
\hline & & LVF & RVF & LVF & RVF & LVF & RVF \\
\hline \multirow{2}{*}{$\begin{array}{c}40 \\
\mathrm{msec}\end{array}$} & LVF & & $7.95^{*}$ & $38.63^{*}$ & & $43.18^{*}$ & \\
\hline & RVF & & & & $46.59^{*}$ & & $57.95^{*}$ \\
\hline 120 & LVF & & & & .00 & 4.54 & \\
\hline msec & RVF & & & & & & $11.36^{*}$ \\
\hline 200 & LVF & & & & & & $6.81 *$ \\
\hline msec & RVF & & & & & & \\
\hline
\end{tabular}

Note-The critical value of $Q(6,33)=5.20$.

only when exposure increased from 40 to $120 \mathrm{msec}$, but not when it increased from 120 to $200 \mathrm{msec}$.

The finding of a significant interaction between exposure duration and visual field while all other factors such as experimental conditions, stimuli, luminance, and task demands were held constant suggests that the relative competence of the two hemispheres varies as a function of the quality of the stimulus representation. The stimuli were identical at all exposure durations, and only the stimulus energy, which determines the spatial-frequency bands that could be extracted, differed. This interaction may then reflect a preferential sensitivity of the hemispheres to different spatial frequencies.

\section{EXPERIMENT 2}

The results of Experiment 1 confirmed the trend observed in other experiments mentioned in the introduction. However, because a between-subject design was used, it could be argued that differences in visual field superiority between the exposureduration conditions may have resulted from sampling errors or particular "strategy" typical to each group. To eliminate this possibly confounding factor, Experiment 1 was replicated using a within-subject design, with two further modifications. First, to avoid a too lengthy experiment with a rather unchallenging task, the faces were presented only in the RVF and LVF and only at 40 and $200 \mathrm{msec}$ (thus eliminating central presentations and the 120 -msec exposure condition). Second, to serve as reference for the third experiment, the test field luminance was set at $10 \mathrm{~mL}$. In pilot work, this value was found to be the more appropriate, since it did not produce too bright a display but did allow for a sufficiently bright display after reduction by a factor of five, as was to be done in Experiment 3.

\section{Method}

The experimental paradigm and equipment were the same as those employed in the previous experiment. The fixation field luminance was kept at $3 \mathrm{~mL}$, while the test field luminance was raised to $10 \mathrm{~mL}$. 
Twelve new male subjects, selected as in Experiment 1, participated in this experiment. Each subject was tested successively under conditions of 40 - and $200-\mathrm{msec}$ exposures. For half the subjects, the 40 -msec condition was conducted first, and for the other half, the 200-msec condition was conducted first. Within each group, the responding hand was counterbalanced. An entire testing session lasted about $35 \mathrm{~min}$.

\section{Results and Discussion}

No reliable effects related to hand responding and to the sex of the faces were observed, and the results were collapsed across these variables. The error rate was $4.1 \%$, and no significant effects were obtained.

A three-way analysis of variance was performed on the mean correct RTs, with one independent factor, order of conditions, and two repeated factors, visual field and exposure duration. Latencies were longer in the first condition tested than in the second (513 and $502 \mathrm{msec}$, respectively), but this difference was not significant $(F<1)$. This variable did not interact with the other two variables, and the mean correct RTs averaged across subjects and order of conditions are given in Table 3 as a function of exposure duration and visual field. Each mean cell is based on 960 correct responses.

Latencies were shorter at 200 -msec exposure than at 40-msec exposure (498 and $517 \mathrm{msec}$, respectively), but this difference was not significant $[F(1,10)=2.72$, $p>.10]$. The only effect to reach significance was the interaction between exposure duration and visual field $[F(1,10)=11.82, p<.01]$. The subjects categorized the faces faster in the LVF than in the RVF at 40-msec exposure, but faster in the RVF than in the LVF at 200-msec exposure. This interaction resulted from an improved performance in RVF presentations when exposure increased from 40 to $200 \mathrm{msec}$, whereas latencies were practically unchanged in LVF presentations at both exposures.

These results confirm the findings of Experiment 1 in showing that the $\mathrm{LH}$ benefits more than the $\mathrm{RH}$ from an increase in stimulus energy as determined by an increase in exposure duration. Taken together, the results of Experiments 1 and 2 suggest that the $\mathrm{LH}$ and the RH are preferentially sensitive to different ranges of spatial frequencies available from the display. In LVF presentations, an improved performance was observed in Experiment 1 when exposure increased from 40 to $120 \mathrm{msec}$ but not when it increased from 120 to $200 \mathrm{msec}$, at a luminance of $3 \mathrm{~mL}$. The higher frequencies that were made available at 200 -msec exposure were thus of no further

Table 3

Mean Latencies in the Two Visual Fields for Each Exposure Duration of Experiment 2

\begin{tabular}{ccc}
$\begin{array}{c}\text { Exposure } \\
\text { Duration }\end{array}$ & LVF & RVF \\
\hline 40 & 504 & 531 \\
200 & 506 & 489 \\
\hline
\end{tabular}

necessity to the $\mathrm{RH}$ for efficient processing. A similar finding was obtained in Experiment 2, in which a higher luminance was used: the increase in stimulus energy from 40 - to 200 -msec presentation time did not result in improved performance in the RH. Conversely, in RVF presentations, both experiments indicate improved performance of the $\mathrm{LH}$ as more stimulus energy was integrated. This suggests that the higher frequencies made available in such conditions could be used by the LH to enhance its processing efficiency.

This differential hemispheric sensitivity to spatial frequency spectral components may then determine the amount of energy to be integrated before cognitive processing of incoming information unfolds within each hemisphere. Since low frequencies are extracted and integrated faster in the visual sensory areas, the greater sensitivity of the $\mathrm{RH}$ to low frequencies may allow this hemisphere to start processing earlier than the $\mathrm{LH}$. In contrast, the greater sensitivity of the LH to higher frequency spectral components would make it require a longer summation of energy before this hemisphere could efficiently process information on which it was better equipped to operate.

This is consistent with Eriksen and Schultz's (1979) continuous flow conception of visual information processing, which suggests a gradual accumulation of information in the visual system, with an increasingly more detailed or exact sensory output over time as energy is integrated in the primary visual area. Thus, although the RH may begin its processing earlier than the LH, the lower resolution of the information on which the RH operates may slow down its cognitive processing and limit its efficiency when demanding discriminations are required. On the other hand, provided the viewing conditions are such as to allow for the extraction and integration of higher frequency components, the higher acuity of the information with which the LH preferentially deals may facilitate its cognitive operations as compared with the RH. This interpretation is also consistent with the results obtained by Hellige (1980) in a memory search task using Sternberg's (1969) additive model. Hellige observed a smaller intercept value for LVF than for RVF presentations, suggesting a faster encoding in the RH. However, the slope was steeper in LVF than in RVF presentations, indicating slower cognitive processing in the $\mathrm{RH}$ than in the LH.

\section{EXPERIMENT 3}

The two previous experiments showed a shift in visual field advantage with an increase in exposure duration while keeping luminance constant within each experiment. This suggests that the amount of energy available from the display, which covaried with variations in presentation time, determined the 
relative efficiency of the hemispheres. Thus, if stimulus energy is the only critical variable, reciprocally varying exposure duration and luminance so as to keep the amount of energy constant should prevent a shift in field superiority from emerging. This assumes that Bloch's law, which was developed by observing visual performance with foveal presentations, holds perfectly in the retinal periphery.

Specifically, the displays were presented at the same exposure durations as in Experiment 2 (40 and $200 \mathrm{msec})$. The same luminance $(10 \mathrm{~mL})$ was used at 40-msec exposure, but a 2-mL luminance was used at 200 -msec exposure, thus keeping energy constant as exposure duration varied. Since presentation conditions at $40 \mathrm{msec}$ (and therefore energy) were to be the same as those prevailing in Experiment 2, which, at this exposure, yielded an LVF advantage, one should now obtain an LVF superiority at both 40 and 200 -msec presentations if the total energy in the display was the critical variable. Alternatively, if the RH and LH need a different amount of energy to efficiently process the information they receive, a pattern of results similar to that found in the previous experiment should be obtained.

\begin{abstract}
Method
The experimental paradigm and equipment were the same as those employed in the previous experiments. The fixation field luminance was kept at $3 \mathrm{~mL}$, while the test field luminance was varied with exposure conditions: at $40-\mathrm{msec}$ presentations, luminance was $10 \mathrm{~mL}$; at 200 -msec presentations, luminance was $2 \mathrm{~mL}$.

Twelve new male subjects, selected as in the previous experiments, participated in this experiment. Order of conditions and responding hand were counterbalanced, as in Experiment 2. An entire experimental session lasted about $35 \mathrm{~min}$.
\end{abstract}

\section{Results and Discussion}

No effect of hand responding and sex of faces reached a reliable level of significance. Error rates were $7.00 \%$. No main effects were significant. The interaction between presentation conditions and visual field was significant $[F(1,11)=5.18, p<.05]$. Differences between the visual fields were observed at 40 -msec exposure $(2.76 \%$ and $4.43 \%$ in the LVF and RVF, respectively) and at $200-\mathrm{msec}$ exposure (3.85\% and $2.97 \%$ in the LVF and RVF, respectively).

A three-way analysis of variance was carried out on the mean correct RTs, with one independent factor, order of presentation, and two repeated factors, presentation conditions and visual field. No main effect reached significance, and the only significant interaction was that between visual field and presentation condition $[F(1,10)=16.13, p<.01]$. The mean correct $R T$ s representing this interaction are shown in Table 4. At 40-msec exposure and $10-\mathrm{mL}$ luminance, latencies were faster in LVF than in RVF presentations ( 517 and $539 \mathrm{msec}$, respectively, $\mathrm{p}<.01$ ), and this result replicates the finding of Experiment 2 under the same conditions. At 200-msec exposure
Table 4

Mean Latencies in the Two Visual Fields for Each Presentation Condition of Experiment 3

\begin{tabular}{ccc}
\hline $\begin{array}{c}\text { Presentation } \\
\text { Condition }\end{array}$ & LVF & RVF \\
\hline $40 \mathrm{msec} / 10 \mathrm{~mL}$ & 517 & 539 \\
$200 \mathrm{msec} / 2 \mathrm{~mL}$ & 540 & 526 \\
\hline
\end{tabular}

and 2-mL luminance, latencies were faster in RVF than in LVF presentations ( 526 and $540 \mathrm{msec}$, respectively, $\mathrm{p}<.05$ ). Although both presentation conditions yielded the same amount of luminous energy and latencies of the same order overall $(528 \mathrm{msec}$ at $40 \mathrm{msec}$ and $533 \mathrm{msec}$ at $200 \mathrm{msec}, \mathrm{F}<1$ ), the two hemispheres did not perform similarly. If the total amount of energy were the only critical variable, the same pattern of results should have prevailed in both presentation conditions. The results may therefore indicate that the duration over which energy is integrated may also be a determinant in the emergence of a visual field effect, and they suggest that processing of the sensory output may be activated at different times in each hemisphere. As noted earlier, the RH may begin its processing before the total summation of energy is completed, at a time when the information is not necessarily the more compatible with an efficient operation but is already sufficient. When energy is integrated over a relatively long duration, the RH may start processing at early stages of information accumulation, which would provide essentially low resolution information and thus make its operation slower. This may also be suggested by the larger number of errors in LVF presentations at a 200-msec exposure duration. This early RH engagement in processing may not be as disrupted when energy is integrated over a brief duration. Conversely, the LH was still found to benefit more than the RH from an increase in exposure duration, and the results may confirm a differential hemispheric sensitivity to spatial frequencies of the stimulus.

Although this interpretation may account for the present findings, the role of the time component in energy summation will have to be further investigated, using more variations in stimulus energy. Moreover, the assumption that Bloch's law holds perfectly in the retinal periphery needs to be empirically examined. Kerr (1971) observed that increases in luminance produced less improvement in visual acuity for targets presented in the periphery than for those presented foveally. Thus, the two presentations in this experiment may not have yielded equal amounts of energy.

\section{GENERAL DISCUSSION}

\section{Emergence of Hemispheric Asymmetries}

The results of these experiments may support the hypothesis that the cerebral hemispheres are pref- 
erentially sensitive to different spatial frequency spectral components of a stimulus. Considering visual stimuli in terms of physical characteristics whose neural correlates are extracted and integrated during early sensory processing may allow one to examine the respective competence of the $\mathrm{LH}$ and $\mathrm{RH}$ in dealing with these characteristics beyond the sensory areas.

The resolving power of the visual system increases with enhancement in stimulus energy, and, as the integration of visual information unfolds, increasingly higher frequency output is available for further processing. The repeatedly observed RH superiority at very brief exposure may then suggest that the $\mathrm{RH}$ needs less energy, and consequently a lower level of resolution, than the $\mathrm{LH}$ to perform its processing, whereas the LH appears to be more efficient in conditions that allow for the extraction of high spatial frequencies.

The slower RTs at $40 \mathrm{msec}$ in Experiment 1 suggest that the lower the spatial frequencies, the slower the processing as a result of the degraded available information. This slower processing occurred despite the fact that low frequencies are extracted and integrated more rapidly than higher frequencies in the visual cortex (Williamson et al., 1978). Thus, what was slowed down when a low level of energy was displayed was the "cognitive" processing taking place beyond the sensory areas, and in such conditions the RH proved to be better equipped than the $\mathrm{LH}$ to perform its processing.

This does not mean, however, that the $\mathrm{RH}$ is the most efficient at the lowest frequency level, at least in this type of categorization task. In fact, a comparison of RTs at 40- and 120-msec exposures in Experiment 1 showed that performance improved with higher energy. Further increase in energy, at the 200-msec exposure, did not improve processing efficiency for the RH in Experiments 1 and 2, but did for the LH. This may indicate that the higher frequencies that could be extracted at $200-\mathrm{msec}$ exposure benefited only the $\mathrm{LH}$, as shown by its faster processing at such an exposure. This suggests that the range of spatial frequencies to which the $R H$ is better tuned may extend from the lowest to an intermediate value in the power spectrum, whereas the LH may operate more efficiently on intermediate-tohigh spatial frequencies. This is similar to a previous suggestion (Sergent, 1982a; Sergent \& Bindra, 1981) that the LH needs a clear and complete visual representation to perform efficiently, and that it is more disrupted than the RH when degraded and partial information is available for processing.

The superiority of one hemisphere over the other in a given task may then depend on the characteristics of the stimulus that are made available through the conditions of presentation. However, this is not the only determining factor, and the requirements of a given task, in terms of the stimulus characteristics that must be processed to perform efficiently, must also be considered (cf. Garner, 1978; Sergent \& Bindra, 1981). In the present experiments, the categorization of faces into "female" and "male" may have made specific demands as to the features that must be examined to make a decision, and these demands may also be expressed in terms of spatial frequencies (cf. Sergent, 1982b). For example, if the categorization had been between faces and pencils, a decision could have been made efficiently even when only low frequencies were available. That is, it would not be necessary to examine specific facial features before deciding that the stimulus was a face and not a pencil, and higher frequencies of the stimuli would not be useful in making the decision. However, when one has to distinguish a female face from a male one, very low frequencies may allow only for perceiving that it is a face, and frequencies of higher bandwidths may be needed to detect the particular features that make female and male faces different.

This may then explain why, in conditions of low acuity, cognitive processing took longer than when information about more distinctive features could be integrated. With more energy, the more relevant distinctive male and female facial features became clearer, which reduced the duration of processing. The fact that this was accompanied by a shift in hemispheric superiority points to the respective efficiency of the hemispheres as a function of the spatial frequencies, not only available for processing, but also required for efficient processing. Thus, the RH may prove to be superior to the $\mathrm{LH}$, in relative terms, when essentially low frequencies can be integrated or when low frequencies are adequate, given the nature of the task.

This may help to explain how one has come to think that the $\mathrm{RH}$ is specialized in processing faces. In tachistoscopic studies on face recognition, the stimuli are often presented for a very brief time, making the higher frequencies less prominent and favoring the $\mathrm{RH}$ in the processing of the stimuli. Most of the tasks used are delayed-matching of fairly dissimilar faces (cf. Sergent \& Bindra, 1981), which may not require that the fine facial features, contained in the higher frequencies, be compared. Moreover, Harmon (1973) and Tieger and Ganz (1979) have shown that low spatial frequencies were sufficient for efficient and accurate face recognition. Thus, facial recognition may be achieved essentially by processing the low frequencies either because the higher frequencies are not available due to low energy or because the low frequencies are sufficient to reach a decision when the faces are fairly dissimilar.

This suggests that the RH does have an advantage over the LH in processing faces, not because it contains a specific "face processor," but because of its 
better tuning to the low range of spatial frequencies. However, as noted earlier, when longer durations are used and when the faces are either fairly similar (Patterson \& Bradshaw, 1975; Sergent, 1982a) or are unexpected well-known physiognomies to be identified (Marzi \& Berlucchi, 1977), the LH becomes superior to the $\mathrm{RH}$, because the high frequencies contained in the face must now be processed to reach an accurate decision. There may thus be no definite specialization of the RH to process faces, and both hemispheres may be involved in facial processing, each in its own way. This is confirmed by the fact that all cases of prosopagnosia (a specific inability to recognize familiar faces) for which an autopsy was performed have been found to suffer from bilateral posterior lesions (Meadows, 1974).

It is noteworthy that most experiments with faces have used black and white photographs whose different shades of gray may reduce the contrast level, making the higher frequencies longer to integrate (Breitmeyer \& Ganz, 1977; Kitterle \& Corwin, 1979). Conversely, experiments with faces reporting an RVF superiority have used line drawings of physiognomies, yielding maximum contrast (Fairweather, Brizzolara, Tabossi, \& Umiltà, 1982; Patterson \& Bradshaw, 1975; Sergent, 1982a), or long-exposed (at least $200 \mathrm{msec}$ ) photographs (Experiments 1, 2, and 3; Jones, 1980; Marzi \& Berlucchi, 1977).

All these considerations suggest that a particular visual field superiority obtained in an experiment is overdetermined by a multitude of factors, none of which in itself is sufficient to account for the finding. However, looking at these factors in terms of input characteristics available or required for processing may reconcile their apparent diversity. The results of the present experiments may also indicate that a large number of tachistoscopic studies may have little validity as to their conclusions, since an opposite outcome could have been obtained had either a longer or a shorter exposure duration been used or a different level of luminance been employed. This may specifically apply to recognition accuracy studies which typically use a low level of energy to produce a fair number of errors. ${ }^{5}$

\section{Conclusion}

The results of these experiments provide further evidence that the cerebral hemispheres may differ in their capacity to process information of low and high sensory resolution (Sergent, 1982b). Such a hypothesis is not meant to "replace" the more traditional views of cerebral lateralization, but rather to explain these dichotomies and to provide a frame of reference at a level that is more compatible with an understanding of cerebral processes. The main suggestion is that the LH preferentially operates on, and produces, high-resolution information, whereas the RH electively deals with, and yields, low-resolution information, in the visual as well as in the auditory, tactual, and motor modalities (see Sergent, Note 2). This implies that both hemispheres can process verbal and visuospatial information, analytically or holistically, but that each hemisphere is constrained by processing limitations and predispositions, depending on the level of resolution available or required in these processes. The brain may then be understood as a single organism whose hemispheres are working together but are preferentially sensitive to different aspects of the same information, the RH being more competent in early processing and the $\mathrm{LH}$ being more involved in refined operations. Thus, the verbal/visuospatial (Milner, 1971) and the analytic/ holistic (Bradshaw \& Nettleton, 1981) dichotomies may represent only approximate translations, in the cognitive language, of a hemispheric difference at the neural level.

\section{REFERENCE NOTES}

1. Sergent, J. How to bully the brain into a right hemisphere superiority in verbal tasks. Paper presented at the Canadian Psychological Association meeting, Montreal, June 1982.

2. Sergent, J. The role of the input in visual hemispheric asymmetries. Manuscript submitted for publication, 1981.

\section{REFERENCES}

Berkley, M. A., Kitterle, F., \& Watkins, D. W. Gratings visibility as a function of orientation and retinal eccentricity. Vision Research, 1975, 15, 239-244.

Bradshaw, G. J., Hicks, R. E., \& Rose, B. Lexical discrimination and letter-string identification in the two visual fields. Brain and Language, 1979, 8, 10-18.

Bradshaw, J. L., \& Nettleton, N. C. The nature of hemispheric specialization in man. The Behavioral and Brain Sciences, 1981, 4, 51-91.

Breitmeyer, B. Simple reaction time as a measure of the temporal properties of the transient and sustained channels. Vision Research, 1975, 15, 1411-1412.

Breitmeyer, B., \& Ganz, L. Temporal studies with flashed gratings: Inferences about human transient and sustained channels. Vision Research, 1977, 17, 861-865.

Bryden, M. P. Tachistoscopic recognition, handedness, and cerebral dominance. Neuropsychologia, 1965, 3, 1-8.

Camprell, F. W., \& Robson, J. G. Application of Fourier analysis to the visibility of gratings. Journal of Physiology, $1968,197,551-566$.

Cohen, G. Hemispheric differences in a letter classification task. Perception \& Psychophysics, 1972, 11, 139-142.

Davis, R., \& Schmit, V. Visual and verbal coding in the interhemispheric transfer of information. Acta Psychologica, 1973, 37, 229-240.

Di Lollo, V. Hemispheric symmetry in visible persistence. Perception \& Psychophysics, 1981, 29, 21-25.

Eriksen, C. W., \& Schultz, D. W. Temporal factors in visual information processing. In J. Requin (Ed.), Attention and performance VII. New York: Academic Press, 1978.

Eriksen, C. W., \& Schultz, D. W. Information processing in visual search: A continuous flow conception and experimental results. Perception \& Psychophysics, 1979, 25, 249-263.

Fairwe ather, H., Brizzolara, D., Tabossi, P., \& Umiltà, C. 
Functional cerebral lateralisation: Dichotomy or plurality? Cortex, 1982, in press.

Flavell, J., \& Draguns, J. A microgenetic approach to perception and thought. Psychological Bulletin, 1957, 54, 197-217.

FukUda, Y., \& Stone, J. Retinal distribution and central projections of Y-, X-, and W-cells of the cat's retina. Journal of Physiology (London), 1974, 37, 749-772.

Garner, W. R. Aspects of a stimulus: Features, dimensions, and configurations. In E. Rosch \& B. B. Lloyd (Eds.), Cognition and categorization. Hillsdale, N.J: Erlbaum, 1978.

Harmon, L. D. The recognition of faces. Scientific American, $1973,227,71-82$.

Harwe rth, R. S., Boltz, R. L., \& Smith, E. L., III. Psychophysical evidence for sustained and transient channels in the monkey visual system. Vision Research, 1980, 20, 15-22.

HaRwe RTH, R. S., \& Levi, D. M. Reaction time as a measure of suprathreshold grating detection. Vision Research, 1978, 18, 1579-1586.

Hellige, J. B. Effects of perceptual quality and visual fields of probe stimulus presentation on memory search for letters. Journal of Experimental Psychology: Human Perception and Performance, 1980, 6, 639-651.

Hellige, J. B., \& Websten, R. Case effects in letter-name matching: A qualitative visual field difference. Bulletin of the Psychonomic Society, 1981, 17, 179-182.

JoNES, B. Sex and handedness as factors in visual-field organization for a categorization task. Journal of Experimental Psychology: Human Perception and Performance, 1980, 6, 494-500.

KAHNEMAN, D. Temporal summation in an acuity task at different energy levels-A study of the determinants of summation. Vision Research, 1964, 4, 557-566.

Kahneman, D., \& Norman, J. The time-intensity relation in visual perception as a function of observer's task. Journal of Experimental Psychology, 1964, 68, 215-220.

KERR, J. L. Visual resolution in the periphery. Perception \& Psychophysics, 1971, 9(3B), 375-378.

Kimura, D. Cerebral dominance and the perception of verbal stimuli. Canadian Journal of Psychology, 1961, 15, 166-171.

KinsBourne, M. If sex differences in brain lateralization exist, they have yet to be discovered. The Behavioral and Brain Sciences, 1980, 3, 241-242.

Kitte rle, F. L., \& Corwin, T. R. Enhancement of apparent contrast in flashed sinusoidal gratings. Vision Research, 1979, 19, 33-39.

KLAtsky, R. L., \& Atkinson, R. E. Specialization of the cerebral hemispheres in scanning for information in short-term memory. Perception \& Psychophysics, 1971, 10, 335-338.

LEEHEY, S. C., \& CAHN, A. Lateral asymmetries in the recognition of words, familiar and unfamiliar faces. Neuropsychologia, $1979,17,619-627$.

Lennie, P. Parallel visual pathways: A review. Vision Research, $1980,20,561-594$.

LiE, I. Visual detection and resolution as a function of retinal locus. Vision Research, 1980, 20,967-974.

Maffei, L., \& Fiorentini, A. Spatial frequency rows in the striate visual cortex. Vision Research, 1977, 17, 357-364.

Marzi, C. A., \& Berlucchl, G. Right visual field superiority for accuracy of recognition of famous faces in normals. Neuropsychologia, 1977, 15, 751-756.

McGlone, J. Sex differences in human brain asymmetry: A critical survey. The Behavioral and Brain Sciences, 1980, 3, 215-263.

Meadows, J. C. The anatomical basis of prosopagnosia. Journal of Neurology, Neurosurgery, and Psychiatry, 1974, 37, 489-501.

MiLnE R, B. Interhemispheric differences in the localization of psychological processes in man. British Medical Bulletin, 1971, 27, 272-277.

Moscovitch, M., Scullion, D., \& Christie, D. Early vs. late stages of processing and their relation to functional hemispheric asymmetries in face recognition. Journal of Experimental Psychology: Human Perception and Performance, 1976, 2, 401-416.

OLDFIELD, R. C. The assessment and analysis of handedness: The Edinburgh inventory. Neuropsychologia, 1971, 9, 97-113.

Patterson, K., \& Bradshaw, J. L. Differential hemispheric mediation of nonverbal visual stimuli. Journal of Experimental Psychology: Human Perception and Performance, 1975, 1, 246-252.

Pring, T. R. The effects of stimulus size and exposure duration on visual field asymmetries. Cortex, 1981, 17, 227-240.

Riggs, L. A. Visual acuity. In J. W. Kling \& L. A. Riggs (Eds.), Experimental psychology. New York: Holt, Rinehart \& Winston, 1971.

RiJsdiJK, J. P., Kroon, J. N., \& van der Wildt, G. J. Contrast sensitivity as a function of position on the retina. Vision Research, 1980, 20, 235-241.

Rizzolatti, G., \& Buchtel, H. A. Hemispheric superiority in reaction time to faces: A sex difference. Cortex, 1977, 13, 300305.

Schiller, P. H., Finlay, B. L., \& Volman, S. F. Quantitative studies of single cell properties in monkey striate cortex, III: Spatial frequency. Journal of Neurophysiology, 1976, 39, 13341351.

Sergent, J. About face: Left-hemisphere involvement in processing physiognomies. Journal of Experimental Psychology: Human Perception and Performance, 1982, 8, 1-14. (a)

Sergent, J. The cerebral balance of power: Confrontation or cooperation? Journal of Experimental Psychology: Human Perception and Performance, 1982, 8, 253-272. (b)

Sergent, J., \& Bindra, D. Differential hemispheric processing of faces: Methodological considerations and reinterpretation. Psychological Bulletin, 1981, 89, 541-554.

Sternberg, S. Memory scanning: Mental processes revealed by reaction time experiments. American Scientist, 1969, 57, $421-457$.

Stone, J., Dreher, B., \& Leventhal, A. Hierarchical and parallel mechanisms in the organization of the visual cortex. Brain Research Reviews, 1979, 1, 345-394.

TIEGER, T., \& GANz, L. Recognition of faces in the presence of two-dimensional sinusoidal masks. Perception \& Psychophysics, 1979, 26, 163-167.

TolmuRst, D. J. Sustained and transient channels in human vision. Vision Research, 1975, 15, 1151-1155.

Tootell, R. B., Silverman, M. S., \& De Valois, R. L. Spatial frequency columns in primary visual cortex. Science, 1981, 214, 813-815.

Williamson, S. J., Kaufman, L., \& Brenner, D. Latency of the neuromagnetic response of the human visual cortex. Vision Research, 1978, 18, 107-110.

Wright, M. J., \& IkedA, H. Processing of spatial and temporal information in the visual system. In F. O. Schmitt \& F. G. Worden (Eds.), The neurosciences: Third Study Program. Cambridge, Mass: M.I.T. Press, 1974.

\section{NOTES}

1. Longer exposures have been used in several experiments, nonetheless yielding visual field asymmetries. In reaction time studies, it has been argued (e.g., Klatsky \& Atkinson, 1971; Moscovitch, Scullion, \& Christie, 1976; Sergent, 1982) that latencies are measured at the onset of exposure, when the stimulus is properly projected to one hemisphere, and that, if both hemispheres received the information, laterality effects should not be observed, but they are. When accuracy is the dependent variable, making response latency nonrelevant, eye movements are usually monitored to ensure that each trial is performed under steadiness of fixation (e.g., Marzi \& Berlucchi, 1977).

2. Although no such recordings are possible in humans, similar physiological organization in simian and human visual systems is 
suggested by the very high correlation between psychophysical responses by monkey and man (Harwerth, Boltz, \& Smith, 1980).

3. Lateral presentation, which is inherent in these tachistoscopic experiments, also bears implications in this respect. Visual acuity drops off sharply with increase in retinal eccentricity, even within the fovea (Riggs, 1971). The number of cones and of sustainedcell receptive fields markedly decreases away from the fovea (Fukuda \& Stone, 1974). This suggests not only that a longer period of energy summation would be required in order to compensate for the decreased acuity (Eriksen \& Schultz, 1978), but also that lateral tachistoscopic presentation does not engage the full perceptual capacities of the subject.

4. The focus of neuropsychologists on "abstract categories" to account for cerebral lateralization may result from their primary goal which is to infer the locus and side of lesions from behavioral and cognitive deficits. Clinicians are concerned with symptoms and not with the underlying mechanisms, favoring the "psychological" at the expense of the "neural." Yet neural problems should be addressed in terms of neural units, and little progress will be made as long as neural mechanisms are interpreted in terms of psychological concepts.

5. The retinal eccentricity at which stimuli are presented has also been found to influence visual field superiority effect. Sergent (Note 1) used the Posner paradigm to investigate this issue and presented pairs of letters either 3 or $11 \mathrm{deg}$ away from fixation. A significant interaction of eccentricity by visual field was obtained, $\mathrm{RH}$ performance being essentially unchanged at both eccentricities and LH performance being markedly lower at large eccentricity.

(Manuscript received July 15, 1981;

revision accepted for publication February 2, 1982.) 